\title{
Fibroblast mitogenic activity of lung lavage fluid from infants with chronic lung disease of prematurity
}

\author{
A E Currie, M Kelly, J R Vyas, H Pandya, D Field, S Kotecha
}

Arch Dis Child Fetal Neonatal Ed 2002;86:F193-F197

See end of article for authors' affiliations

Correspondence to:

Dr Kotecha, Department of

Child Health, University of

Leicester, Leicester LE2 7LX,

UK; sk43@@le.ac.uk

Accepted

18 December 2001

Background: Lung fibrosis is thought to be important in chronic lung disease of prematurity (CLD). Methods: Fibroblast proliferative activity was assessed in 207 bronchoalveolar lavage fluid (BALF) samples from 43 infants. Sixteen developed CLD (birth weight $765 \mathrm{~g}$ (630-1230), gestation 26.5 weeks (23-29)), 18 developed respiratory distress syndrome (RDS) (birth weight $1415 \mathrm{~g}$ (430-4 160), gestation 31 weeks (23-39)), and nine control infants (birth weight $2110 \mathrm{~g}$ (900-3720), gestation 32 weeks (26-41)) received mechanical ventilation for non-pulmonary reasons.

Results: The fibroblast proliferative activity relative to $10 \%$ fetal calf serum was $64-75 \%$ in infants with CLD, $55-86 \%$ in the RDS group, and $42-68 \%$ in control infants during the first 5 weeks of life. Only at day 3 was there a difference between the groups (CLD 72\% v control 42\%, p $<0.01$; RDS 63\% v control $42 \%, p<0.05)$. With the use of neutralising antibodies, platelet derived growth factor $\mathrm{BB}$ (PDGF-BB) and epidermal growth factor were undetectable, and insulin-like growth factor I (IGF-I) accounted for $14 \%(p<0.05)$ and $11 \%(p<0.005)$ of BALF mitogenic activity from the RDS and CLD groups respectively.

Conclusions: The mitogenic activity of BALF was similar in the three groups studied and was only partially accounted for by IGF-I. Growth factors other than PDGF-BB and IGF-I contribute significantly to this process.

A lthough the risk factors for chronic lung disease of prematurity (CLD) have been accurately described, the underlying pathogenesis is less clear. ${ }^{2}$ Oxygen toxicity and barotrauma are important risk factors, and a more recently described addition is pulmonary inflammation. ${ }^{3}$ Histologically, in infants who die from CLD, lung fibrosis is a characteristic feature of the disease. ${ }^{4}$ The hyaline membranes, which are present early in the development of neonatal respiratory distress syndrome (RDS), are replaced during the second week by interstitial and perialveolar fibrosis. The septal walls are thickened, and myofibroblast proliferation is seen by electron microscopy. ${ }^{45}$ Using immunohistochemistry, we have shown that newly synthesised collagen, namely procollagen type I, is deposited early in infants who die from respiratory failure compared with those who die from nonrespiratory causes. ${ }^{6}$ Components of the extracellular matrix, including $\mathrm{N}$-termini of collagen molecules and fibronectin, are also increased in tracheal fluid obtained from infants who develop CLD. ${ }^{78}$ Thus pulmonary fibrosis appears to be a hallmark of CLD.

Development of pulmonary fibrosis is dependent on the proliferation of fibroblasts together with synthesis and deposition of the extracellular matrix by these cells. ${ }^{9}$ Proliferation of fibroblasts and the subsequent synthesis of the extracellular matrix is thought to be mediated by growth factors. Transforming growth factor $\beta$ (TGF- $\beta$ ) is a potent profibrotic agent promoting the synthesis and deposition of the extracellular matrix. ${ }^{10}$ We have previously shown that it is considerably increased early in bronchoalveolar lavage fluid (BALF) obtained from infants who developed CLD compared with those infants who did not. ${ }^{11}$ The growth factors platelet derived growth factor B (PDGF-BB) and insulin-like growth factor (IGF-I) are potent mitogens for fibroblasts. ${ }^{12}{ }^{13}$

IGF-I consists of B and A chains, with the mature 70 amino acid molecule being highly conserved in vertebrates, showing $40 \%$ homology with insulin and $60 \%$ with IGF-II. ${ }^{12}$ IGF-I is expressed in fetal tissues by mesenchymal cells, and its ablation in mice results in decreased prenatal growth and lethality immediately after birth. ${ }^{14}$ Postnatally, IGF-I has multiple functions, including acting as a progression factor for fibroblasts, thus inducing their proliferation. IGF-I has been shown to be increased in systemic sclerosis and in idiopathic pulmonary fibrosis. ${ }^{15}{ }^{16}$ PDGF is a $30 \mathrm{kDa}$ protein of either homodimer (PDGF-AA or PDGF-BB) or a heterodimer (PDGF-AB) ${ }^{13}$ Dimers with at least one $\mathrm{B}$ chain will bind to the receptor PDGFR- $\beta$. PDGF-BB is more relevant to respiratory biology as it is a potent mitogen for lung fibroblasts and has been shown to be increased in respiratory diseases such as idiopathic lung fibrosis ${ }^{17}$ and acute lung injury. ${ }^{18}$ Sources within the lung include alveolar macrophages, which are increased in many respiratory diseases including CLD. ${ }^{19}$

As both IGF-I and PDGF-BB are potent mitogens for lung fibroblasts, and as lung fibrosis is a hallmark of CLD, we postulated that BALF obtained from infants who developed CLD would have greater fibroblast mitogenicity than similar fluid obtained from infants who required mechanical ventilation for neonatal RDS and for non-pulmonary reasons. In addition, by using antibody neutralising experiments, we determined whether PDGF-BB or IGF-I contributed to the fibroblast proliferative activity of BALF.

\section{METHODS}

\section{Patient groups}

Three groups of mechanically ventilated infants were studied: (a) CLD group: infants who developed RDS followed by CLD - that is, who were oxygen dependent at 28 days of age and had an abnormal chest radiograph; $(b)$ RDS group:

Abbreviations: CLD, chronic lung disease of prematurity; RDS, respiratory distress syndrome; TGF, transforming growth factor; BAL, bronchoalveolar lavage; BALF, bronchoalveolar lavage fluid; PDGF-BB, platelet derived growth factor B; IGF-I, insulin-like growth factor I; FCS, fetal calf serum; FGM, fibroblast growth medium; EGF, epidermal growth factor 
Table 1 Patient characteristics

\begin{tabular}{|c|c|c|c|}
\hline & CLD & RDS & Control \\
\hline Number & 16 & 18 & 9 \\
\hline Male:female & $9: 7$ & $11: 7$ & $6: 3$ \\
\hline Birth weight $(g)$ & 765 (630-1230) & $1415(430-4160)$ & $2110(900-3720)$ \\
\hline Gestational age (weeks) & $26.5(23-29)$ & $31(23-39)$ & $32(26-41)$ \\
\hline Antenatal dexamethasone & $15(94 \%)$ & $8(44 \%)$ & $3(33 \%)$ \\
\hline Surfactant administration & $15(94 \%)$ & $15(83 \%)$ & $1(11 \%)$ \\
\hline Day 1 maximum peak inspiratory pressure $\left(\mathrm{cm} \mathrm{H}_{2} \mathrm{O}\right)$ & $17(14-22)$ & $19(14-29)$ & $15(14-20)$ \\
\hline Day 1 maximum $\mathrm{FiO}_{2}$ & $0.40(0.24-0.90)$ & $0.41(0.21-1.00)$ & $0.24(0.21-0.28)$ \\
\hline Day 1 maximum mean airway pressure $\left(\mathrm{cm} \mathrm{H}_{2} \mathrm{O}\right)$ & $7.5(5-21)$ & $9.5(4.4-29)$ & $5.3(4-7)$ \\
\hline Day 1 maximum oxygenation index & $5.3(2.1-30.5)$ & 5.9 (0.9-94.3) & $1.8(1.3-3.0)$ \\
\hline
\end{tabular}

infants who developed RDS but were nursed in air and had a normal chest radiograph by day 28 of age; (c) control group: infants who received mechanical ventilation for nonpulmonary reasons and had an oxygen requirement of $\leqslant 28 \%$ throughout the study period.

Infants of mothers who had infection or prolonged rupture of membranes (of $>48$ hours) were excluded, as were infants with documented or strongly suspected sepsis-that is, with positive blood or endotracheal tube secretion cultures or raised white cell count or $\mathrm{C}$ reactive protein concentration $(>0.5$ $\mathrm{mg} / \mathrm{l})$.

\section{Bronchoalveolar lavage (BAL)}

BAL was performed at the time of clinically indicated tracheal suctioning as previously described. ${ }^{11}{ }^{20-22}$ Briefly, the infant was placed in the supine position with the head turned to the left to direct the catheter into the right lower lobe. An FG 5 catheter was gently inserted in a segmental bronchus until resistance was felt, and two aliquots of $1 \mathrm{ml} / \mathrm{kg}$ saline was instilled. With the catheter in situ, after two or three ventilator breaths, the instilled fluid was aspirated back with a suction pressure of 5-7 kPa. The procedure was performed at the time of clinically indicated routine suctioning. The first BAL was performed within 24 hours of birth. Thereafter the infants underwent BAL twice a week until 35 days of age or extubation, whichever occurred earlier. The local research ethics committee had approved the lavage procedure, and informed parental consent was obtained before the procedure was started. The collected BALF was placed on ice and centrifuged at $500 \mathrm{~g}$ at room temperature for 10 minutes within 10 minutes of collection. The supernatant was stored at $-70^{\circ} \mathrm{C}$ until fibroblast assays were performed.

\section{Fibroblast proliferation assays \\ Cell culture}

Swiss $3 \mathrm{~T} 3$ neonatal mouse lung fibroblasts (European Collection of Cell Cultures) were maintained in Dulbecco's modification of Eagle's medium containing penicillin (100 $\mathrm{U} / \mathrm{ml})$, streptomycin ( $100 \mu \mathrm{g} / \mathrm{ml})$, amphotericin B ( $100 \mu \mathrm{g} / \mathrm{ml})$, sodium pyruvate $(1 \mathrm{mM}), 1 \times$ Glutamax II, and $1 \times$ MEM nonessential amino acids (fibroblast growth medium (FGM)) supplemented with $10 \%$ fetal calf serum (FCS). All tissue culture reagents were supplied by BRL/Life Technologies (Paisley, Scotland, UK). Cell cultures were used between passages 6 and 10. The cells tested negative for mycoplasma contamination.

Microwell fibroblast proliferation cultures

Adherent cells were suspended with $0.02 \%$ trypsin/EDTA, and the reaction quenched with FGM/10\% FCS. After centrifugation, the cells were resuspended in fresh FGM/10\% FCS to give a final concentration of $5 \times 10^{3} / 200 \mu \mathrm{l}$. Then $200 \mu \mathrm{l}$ of this cell suspension was introduced into 96 well plates. After overnight incubation at $37^{\circ} \mathrm{C}$ in $5 \% \mathrm{CO}_{2} / 95 \%$ air to permit cell adherence, the cells were quiesced in FGM/0.5\% FCS for a further 24 hours. Thereafter, cells were incubated under the following experimental conditions: controls of $0.5 \%, 10 \%$ FCS, $20 \mathrm{ng} / \mathrm{ml}$ IGF-I or PDGF-BB; or BALF with or without growth factor and/or antibody (recombinant PDGF-BB, epidermal growth factor (EGF), and IGF-I and respective antibodies; R\&D Systems Europe, Abingdon, Oxon, UK). All experiments were performed in triplicate.

Preliminary experiments showed that 48 hours of incubation was optimal to show fibroblast proliferation. Additional experiments showed that $1: 10$ to $1: 20$ dilution of BALF was optimal to show fibroblast proliferation (see below and fig 3 ). Thus, a 1:10 dilution was used for fibroblast proliferation assays in triplicate, and, because of limited volumes of BALF obtained from preterm infants, a 1:20 dilution was used in triplicate for antibody neutralising experiments.

\section{Methylene blue assay}

The proliferation of Swiss 3T3 cells was determined by a modified assay described by Oliver et al. ${ }^{23}$ After removal of the culture medium, the cells were fixed with $10 \%$ formaldehyde in phosphate buffered saline for one hour, and $100 \mu \mathrm{l} 2 \%$ (w/v) fresh methylene blue in $0.1 \mathrm{M}$ borate buffer $(\mathrm{pH} 8.5)$ was added. After elution of the dye from the cells with $1: 1(\mathrm{v} / \mathrm{v})$ ethanol and $0.1 \mathrm{M} \mathrm{HCl}$, the absorbance was measured at 650 $\mathrm{nm}$ with a microplate photometer (Dynatech Labs). Initial experiments validated the methylene blue assay with both cell counting and incorporation of $\left[{ }^{3} \mathrm{H}\right]$ thymidine. The results of the methylene blue assay were within $10 \%$ of the results obtained by cell counting and $\left[{ }^{3} \mathrm{H}\right]$ thymidine incorporation.

\section{Antibody blocking experiments}

Initial experiments using a dose range of $0-100 \mathrm{ng} / \mathrm{ml}$ recombinant growth factor (PDGF-BB and IGF-I) and the fibroblast proliferation assay described above showed that $20 \mathrm{ng} / \mathrm{ml}$ growth factor (both PDGF-BB and IGF-I) resulted in near maximum fibroblast proliferation. Additional experiments showed that $10 \times$ manufacturer's specified neutralising dose $_{50}$ of the respective antibody completely abolished the mitogenic activity of both growth factors, each used at $100 \mathrm{ng} / \mathrm{ml}$. For experiments using BALF, the lavage fluid was incubated for two hours on ice with the antibody before addition to the fibroblasts. The experiments were terminated after 48 hours of incubation as described above by the addition of $10 \%$ formaldehyde in phosphate buffered saline. Cell proliferation was determined by using the methylene blue assay described above.

\section{Data analysis}

Results are given as median (range) for patient characteristics such as birth weight and gestation, and median (interquartile range) for the fibroblast proliferation assays. All experiments with the fibroblast proliferation assay were performed in triplicate. The mean $\mathrm{A}_{650}$ of the triplicates is given for single experiments. When multiple plates were compared, the $\mathrm{A}_{650}$ 


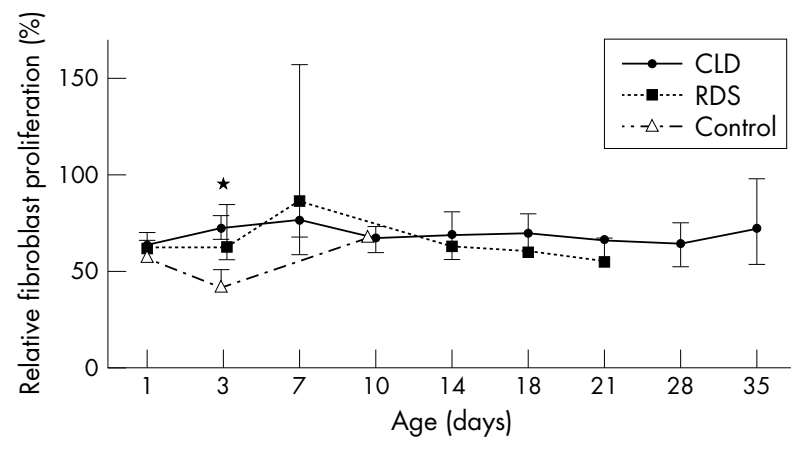

Figure 1 Relative fibroblast proliferation induced by bronchoalveolar lavage fluid obtained from infants who progressed to chronic lung disease of prematurity (CLD), who developed and recovered from neonatal respiratory disease (RDS), and control infants receiving mechanical ventilation for non-respiratory reasons. The proliferative activity is relative to the $A_{650}$ value obtained for $10 \%$ fetal calf serum for each 96 well plate. ${ }^{*} p<0.01$ for CLD $v$ control and $p<0.05$ for RDS $v$ control.

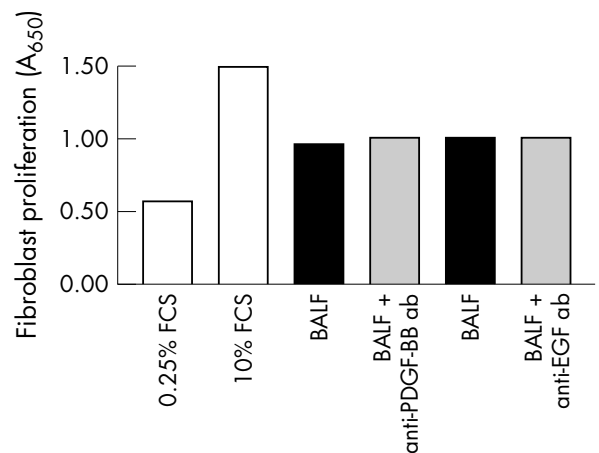

Figure 2 Neutralising antibodies were used against platelet derived growth factor (PDGF-BB) and epidermal growth factor (EGF) to show whether bronchoalveolar lavage fluid (BALF) fibroblast activity was dependent on these two growth factors. Parallel experiments showed that BALF spiked with PDGF-BB did increase fibroblast proliferation, but this was decreased to baseline levels by prior addition of a neutralising antibody (ab) to PDGF-BB.

value for each well was calculated relative to the value for $10 \%$ FCS, which was designated $100 \%$ for each plate. Groups were compared by using the Mann-Whitney U test for unpaired groups, and the Wilcoxon rank test for paired samples (antibody experiments). A p $<0.05$ was considered significant.

\section{RESULTS}

\section{Patient characteristics}

A total of 207 BAL samples were obtained from 43 infants requiring mechanical ventilation. Thirty one BAL samples were excluded because of the small volume, repeated fungal infection with the fibroblast proliferation assay, sepsis, blood staining, or they were from control infants who required $>28 \%$ oxygen. Of the 43 infants studied, 34 developed respiratory failure due to RDS, and, of these, 16 (nine boys, seven girls) progressed to develop CLD, and 18 ( 11 boys, seven girls) recovered and were nursed in air by 28 days of age. Nine required mechanical ventilation for non-pulmonary reasons. The chest radiographs for the infants from the CLD and RDS groups on day l were very similar, showing features of the RDS, but by day 28 of age the chest radiographs for infants in the CLD group had abnormalities, with mild to moderate haziness being the most common finding. No infant had cystic changes on their radiograph. The chest radiographs for the control infants were reported to be normal.

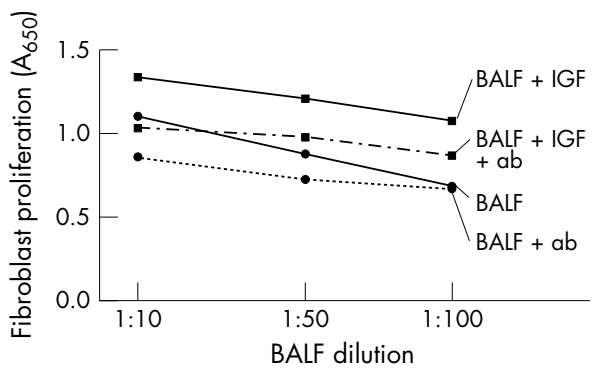

Figure 3 A typical experiment showing that bronchoalveolar lavage fluid (BALF) dilutions increased fibroblast proliferation and that spiking diluted BALF with insulin-like growth factor I (IGF-I) further increased this proliferation and that it was prevented by the prior addition of a neutralising antibody (ab) to IGF-I. Furthermore, this antibody also decreased the fibroblast activity of native BALF at $1: 10$ and $1: 50$ dilutions.

Table 1 shows patient characteristics including use of surfactant and antenatal corticosteroids. In particular, 15/16 (94\%) in the CLD group, $15 / 18$ (83\%) in the RDS group, and $1 / 9(11 \%)$ in the control group were treated with exogenous surfactant. Significant differences in gestation and birth weight were noted between infants in the CLD and RDS groups $(p<0.0005)$ and those in the CLD and control groups $(p<0.0001)$, but not between those in the RDS and control groups (birth weight, $\mathrm{p}=0.13$; gestation, $\mathrm{p}=0.073$ ). Significant differences were also found between the CLD and control groups for inspired oxygen $(p<0.001)$ and oxygenation index $(\mathrm{p}<0.005)$, but not for peak inspiratory pressure in the first 24 hours of age, and between the RDS and control groups for inspired oxygen $(\mathrm{p}<0.01)$ and oxygenation index $(\mathrm{p}<0.01)$. The difference for peak inspiratory pressure in the first 24 hours of age between the RDS and control groups approached significance $(\mathrm{p}=0.06)$.

\section{Fibroblast mitogenic activity of BALF}

Figure 1 shows the results. In the CLD group, the $\mathrm{A}_{650}$ relative to $10 \%$ FCS was $64-75 \%$ during the five week study period. In the infants with RDS, the relative fibroblast proliferation was $55-63 \%$, except for an increase to $86 \%$ on day 7 . In the control group, the values were between $42 \%$ and $68 \%$. Significant differences were only found between the groups at day 3 of age (CLD $v$ controls: $72 \% v 42 \%, \mathrm{p}<0.01$; RDS $v$ controls: $63 \% v$ $42 \%, p<0.05)$, but not between the CLD and RDS groups at any time. Gestation, birth weight, and use of antenatal corticosteroids or exogenous surfactant did not influence the day 1 results for fibroblast proliferation in the different groups studied. (Only day 1 results were compared for gestation, birth weight, and use of antenatal corticosteroids and surfactant because later samples are likely to be influenced by postnatal factors such as oxygen and ventilation).

Detection of fibroblast mitogenic growth factors in BALF Using neutralising antibodies to PDGF-BB and EGF, we were unable to contribute any fibroblast proliferative activity in BALF to these two growth factors (fig 2). Parallel experiments "spiking" BALF with growth factor showed that the increased proliferation of fibroblasts with $20 \mathrm{ng} / \mathrm{ml}$ PDGF-BB was prevented by the prior addition of neutralising antibody to PDGF-BB. As expected, spiking with EGF did not increase fibroblast proliferation (data not shown).

Further experiments used neutralising antibodies to IGF-I to show whether this growth factor contributed to the mitogenic activity observed in BALF from ventilated infants. Fibroblast proliferative activity was shown in BALF by using dilutions of 1:10 to 1:100 (one individual experiment shown in fig 3). Spiking each diluted BALF with IGF-I increased the fibroblast mitogenic activity of the BALF, and this was 


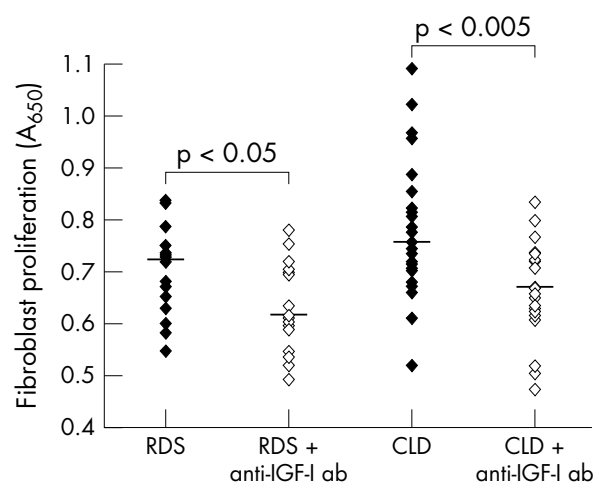

Figure 4 Bronchoalveolar lavage fluid (BALF) from infants in the respiratory distress syndrome (RDS) and chronic lung disease (CLD) groups was used at 1:20 dilution in triplicate to show whether insulin-like growth factor I (IGF-I) contributed significantly to BALF fibroblast proliferative activity. The mitogenic activity for fibroblasts was decreased by $14 \%$ and $11 \%$ respectively in BALF obtained from infants with RDS $(p<0.05)$ and CLD $(p<0.005)$ by the prior addition of the IGF-I neutralising antibody (ab).

decreased by the prior addition of neutralising antibody to IGF-I. Similarly, this antibody decreased the mitogenic activity of BALF. We proceeded to determine whether IGF-I contributed significantly to mitogenic activity in BALF obtained from infants who developed CLD when compared with those who did not. Figure 4 shows the results: using the antibody to IGF-I, the relative fibroblast proliferative activity decreased by $14 \%$ from a median $\mathrm{A}_{650}$ of 0.720 (range $0.552-0.839$ ) to 0.618 (range $0.495-0.782)(\mathrm{p}<0.05)$ in the RDS group, and by $11 \%$ from 0.753 (range $0.524-1.090)$ to $0.673(0.479-0.838)$ in the CLD group $(\mathrm{p}<0.005)$.

\section{DISCUSSION}

We have found that fibroblast proliferative activity of BALF from infants who progressed to CLD and those who developed and recovered from RDS was remarkably similar to that observed in control infants who were more mature. Only at day 3 were differences found between the three groups (fig 1). In addition, antibody neutralising experiments showed that this proliferative activity was not due to PDGF-BB or EGF, and only $11-15 \%$ was attributable to IGF-I. Factors other than these growth factors appear to be responsible for promoting fibroblast proliferation in the lungs of newborn infants.

Pathological studies suggest that lung fibrosis is a prominent feature in the pathogenesis of CLD. Increased fibroblast numbers and increased deposition of components of the extracellular matrix have been observed in these studies. ${ }^{45}$ For lung fibrosis to occur, two events are likely: the fibroblast population may increase by proliferation of these cell types or they may produce increased components of the extracellular matrix. This study aptly refutes the widely held view that fibroblast proliferation is prominent in CLD. More likely is the latter possibility of increased synthesis and deposition of collagen and other components of the lung matrix. Indeed this view is supported by our previous data showing an early increase in TGF- $\beta$ in the lungs of infants who develop $\mathrm{CLD}^{11}$ and an early increase in procollagen in infants who died during the first week of life from respiratory failure. ${ }^{6}$. Other components, especially of collagen, have also been shown to be increased in infants who progress to develop CLD. ${ }^{724}$

In newborn infants, rapid growth and remodelling of the airways is occurring. The same growth factors that are important to the fibrotic process are also thought to play a role in normal lung growth and development, including normal alveolisation. ${ }^{25} 26$ Thus, dysregulated rather than increased profibrotic proliferative activity may be more important in the pathogenesis of CLD. Margraf et $a^{l^{27}}$ and Thibeault et $a^{28}$ have both shown that lung growth in infants with CLD is disordered, resulting in decreased alveolisation, increased mean linear diameter of alveoli, and decreased lung surface area. They also reported disordered deposition of elastin in dense focal areas, which would be expected to affect lung mechanics, including compliance. These observations suggest that normal lung growth is disordered in CLD and that regulation of lung growth, including deposition of components of the extracellular matrix, may be abnormal rather than an increase in numbers of fibroblasts. TGF- $\beta 1$, which we have previously reported to be increased in $\mathrm{CLD}^{11}$ is a known inhibitor of alveolisation ${ }^{29}$ and may be part of a cascade leading to disordered lung growth in CLD. In this study, we did not study the production of components of the extracellular matrix, which may be of greater importance than increased proliferation of fibroblasts.

As rapid lung growth ${ }^{25}{ }^{30}$ is probably regulated by the growth factors we have studied, any additional growth factor activity resulting from repair/remodelling processes after acute lung injury may be difficult to identify. Another explanation for the discrepancy between the increased fibroblast numbers observed at autopsy of infants who have died from CLD when compared with control infants and our data showing minimally increased mitogenicity for fibroblasts may be the removal of fibroblasts from the lung by the process of apoptosis. ${ }^{31}$ Most studies have concentrated on the proliferation of fibroblasts, and yet few have studied the removal of this cell type from the lung. Clearly the balance between fibroblast proliferation and removal of these cells will affect whether or not lung fibrosis occurs.

Growth factors such as IGF-I and PDGF-BB are known mitogens for lung fibroblasts, ${ }^{12}{ }^{13}$ but in BALF from those infants who did and did not develop CLD, we were unable to show any contribution by PDGF-BB, and only $10-15 \%$ activity was attributable to IGF-I. This is unlikely to be due to methodological problems because the increased proliferation caused by spiking the lavage fluid was prevented by the prior addition of the relevant neutralising antibody. The existence of inhibitory autoantibodies to PDGF-BB in BALF has been postulated. We would like to speculate that the lack of fibroblast proliferation caused by PDGF-BB was not due to the presence of inhibitors because the spiking of BALF resulted in fibroblast proliferation, as the presence of inhibitors would have prevented such an effect. Factors other than the growth factors that we have studied are known to promote proliferation of fibroblasts. Particular attention has recently focused on components of the clotting cascades. Thrombin has been shown to be a potent mitogen for fibroblasts and has been shown to be increased in respiratory diseases in adults. ${ }^{32}$

We have used methylene blue to assess the proliferation of fibroblasts with BALF, FCS, and growth factors. It is a simple technique which gives repeatable results. We validated the technique using $\left[{ }^{3} \mathrm{H}\right]$ thymidine incorporation and found a good correlation between the two methods, as previously reported by Laurent's group. ${ }^{23}$ However, we are aware that the staining is proportional to both cell volume and cell numbers, but the correlation between this method and incorporation of thymidine is reassuring. The antibody neutralising experiments were performed with spiking of the BALF with IGF-I and PDGF-BB as well as EGF (as a control). ${ }^{33}$ We found that addition of IGF-I and PDGF-BB increased fibroblast proliferation, but the simultaneous addition of neutralising antibody abrogated this increase thus validating the method.

In summary, we are not aware of any other study showing the fibroblast proliferative activity of BALF obtained from infants who develop CLD. Fibroblast proliferation was remarkably similar in all infants studied, suggesting that fibroblast proliferation is not prominent in the development of CLD. In addition, we have only been able to account for $10-15 \%$ of the mitogenic activity for fibroblasts in lung lavage fluid from newborn infants. Factors other than IGF-I and 
PDGF-BB are responsible for promoting fibroblast division in the newborn lung.

\section{ACKNOWLEDGEMENTS}

A E C and M K were supported by Action Research, and J R V by the British Lung Foundation. We would also like to thank the parents and staff at the neonatal unit at the Leicester Royal Infirmary.

\section{Authors' affiliations}

A E Currie, M Kelly, J R Vyas, H Pandya, D Field, S Kotecha, Department of Child Health, University of Leicester, Leicester LE2 7LX, UK

This publication is dedicated to the memory of Martin Kelly.

\section{REFERENCES}

1 Kotecha S, Silverman M. Chronic respiratory complications of neonatal disorders. In: Landau LI, Taussig LM, eds. Textbook of pediatric respiratory medicine. St Louis, Missouri: Mosby, 1999:464-521.

2 Currie AE, Kotecha S. Chronic lung disease of prematurity. Care of the Critically III 1997; 12:14-17

3 Kotecha S. Cytokines in chronic lung disease of prematurity. Eur J Pediatr 1996;155:514-17.

4 Stocker JT. The respiratory tract. In: Stocker JT, Dehner LP, eds. Pediatric pathology. Philadelphia: JB Lippincott Co, 1992:533-41.

5 Van Lierde S, Cornelis A, Devlieger $\mathrm{H}$, et al. Different patterns of pulmonary sequelae after hyaline membrane disease: heterogeneity of bronchopulmonary dysplasia? A clinicopathological study. Biol Neonate 1991;60:152-62.

6 Kotecha S, Wangoo A, Cook T, et al. Detection and distribution of TGF-beta, procollagen I, and types I and III collagen in the lungs of infants who die from respiratory failure. Am J Respir Crit Care Med 1996:153:A761.

7 Heikinheimo M, Halila R, Marttinen E, et al. N-terminal propeptide of type III collagen in tracheal fluid and serum in preterm infants at risk for bronchopulmonary dysplasia. Pediatr Res 1992;31:340-3

8 Shoemaker CT, Reiser KM, Goetzman BW, et al. Elevated ratios of type $\mathrm{I} / \mathrm{III}$ collagen in the lungs of chronically ventilated neonates with respiratory distress. Pediatr Res 1984;18:1176-80.

9 Coker RK, Laurent GL. Pulmonary fibrosis: cytokines in the balance. Eur Respir J 1998;11:1218-21.

10 Border WA, Noble NA. Transforming growth factor $\beta$ in tissue fibrosis. New Engl J Med 1994;331:1286-92.

11 Kotecha S, Wangoo A, Silverman $M$, et al. Increase in transforming growth factor- $\beta_{1}$ concentration in bronchoalveolar lavage fluid obtained from infants with chronic lung disease of prematurity. J Pediatr 1996; 128:464-9.

12 Reinecke $M$, Collet $C$. The phylogeny of the insulin-like growth factors. Int Rev Cytol 1998;183;1-94.

13 Heldin C-H, Westermark B. Mechanism of action and in vivo role of platelet-derived growth factor. Physiol Rev 1999;79:1283-316.

14 Liu JL, LeRoith D. Insulin-like growth factor I is essential for postnatal growth in response to growth hormone. Endocrinology $1999 ; 140: 5178-84$
15 Harrison NK, Cambrey AD, Myers AR, et al. Insulin-like growth factor-I is partially responsible for fibroblast proliferation induced by bronchoalveolar lavage fluid from patients with systemic sclerosis. Clin Sci 1994;86:141-8.

16 Uh ST, Inove Y, King Jr TE, et al. Morphometric analysis of insulin-like growth factor-l localisation in lung tissues of patients with idiopathic pulmonary fibrosis. Am J Respir Crit Care Med 1998;158:1626-35.

17 Antoniades HN, Bravo MA, Avila RE, et al. Platelet derived growth factor in idiopathic pulmonary fibrosis. J Clin Invest 1990;86:1055-64.

18 Walsh J, Absher M, Kelley J. Variable expression of platelet derived growth factor family proteins in acute lung injury. Am J Respir Cell Mol Biol 1993;9:637-44.

19 Ogden BE, Murphy SA, Saunders GC, et al. Neonatal lung neutrophils and elastase/protease inhibitor imbalance. Am Rev Respir Dis 1984;130:817-21

20 Kotecha S, Chan B, Azam N, et al. Increase in interleukin-8 and soluble intercellular adhesion molecule-1 in bronchoalveolar lavage of premature infants with chronic lung disease. Arch Dis Child Fetal Neonatal Ed 1995;72:F90-6.

21 Kotecha S, Wilson L, Wangoo A, et al. Increase in interleukin-1 beta and interleukin-6 in bronchoalveolar lavage fluid obtained from infants with chronic lung disease of prematurity. Pediatr Res 1996:40:250-6.

22 Kotecha S. Neonatal aspects of bronchoalveolar lavage. In ERS Task Force. Bronchoalveolar lavage in children. Eur Respir J 2000;15:217-31.

23 Oliver MH, Harrison NK, Bishop JE, et al. A rapid and convenient assay for counting cells cultured in microwell plates: application for assessmen of growth factors. J Cell Sci 1989:92:513-18.

24 Ohki Y, Kato M, Kimura H, et al. Elevated type IV collagen in bronchoalveolar lavage fluid from infants with bronchopulmonary dysplasia. Biol Neonate 2001;79:34-8.

25 Kotecha S. Lung growth: implications for the newborn infant. Arch Dis Child Fetal Neonatal Ed 2000;82:F69-74.

26 Groneck P, Gotze Speer B, Oppermann M, et al. Association of pulmonary inflammation and increased microvascular permeability during the development of bronchopulmonary dysplasia: a sequential analysis of inflammatory mediators in respiratory fluids of high-risk preterm neonates. Pediatrics 1994;93:712-18.

27 Margraf LR, Tomashefski JF, Bruce MC, et al. Morphometric analysis of the lung in bronchopulmonary dysplasia. Am Rev Respir Dis $1991 ; 143: 391-400$.

28 Thibeault DW, Mabry SM, Ekekezie II, et al. Lung elastic tissue maturation and perturbations during the evolution of chronic lung disease. Pediatrics 2000;106:1452-9.

29 Serra R, Pelton RW. TGF beta inhibits branching morphogenesis and $\mathrm{N}$-myc expression in lung bud organ cultures. Development 1994; 120:2153-61.

30 Shannon JM, Deterding RR. Epithelial-mesenchymal interactions in lung development. In: MacDonald JA, ed. Lung growth and development. New York: Marcell Decker, 1997:81-1 18.

31 Bruce MC, Honaker CE, Cross RJ. Lung fibroblasts undergo apoptosis following alveolarization. Am J Respir Cell Mol Biol 1999;20:228-36.

32 Hernandez-Rodriguez NA, Cambrey AD, Harrison NK, et al. Role of thrombin in pulmonary fibrosis. Lancet 1995;346:1071-3.

33 Currie AE, Vyas J, MacDonald J, et al. Epidermal growth factor in the lungs of infants developing chronic lung disease. Eur Respir J $2001 ; 18: 796-800$. 